\title{
Palmprint and face score level fusion: hardware implementation of a contactless small sample biometric system
}

\author{
Audrey Poinsot \\ Fan Yang \\ Vincent Brost \\ University of Burgundy \\ Le2i Laboratory \\ Batiment Mirande, Aile de l'Ingenieur, 9 \\ BP 400, Dijon, 21000 France \\ E-mail: audrey.poinsot@u-bourgogne.fr
}

\begin{abstract}
Including multiple sources of information in personal identity recognition and verification gives the opportunity to greatly improve performance. We propose a contactless biometric system that combines two modalities: palmprint and face. Hardware implementations are proposed on the Texas Instrument Digital Signal Processor and Xilinx Field-Programmable Gate Array (FPGA) platforms. The algorithmic chain consists of a preprocessing (which includes palm extraction from hand images), Gabor feature extraction, comparison by Hamming distance, and score fusion. Fusion possibilities are discussed and tested first using a bimodal database of 130 subjects that we designed (uB database), and then two common public biometric databases (AR for face and PolyU for palmprint). High performance has been obtained for recognition and verification purpose: a recognition rate of $97.49 \%$ with AR-PolyU database and an equal error rate of $1.10 \%$ on the $\mathrm{uB}$ database using only two training samples per subject have been obtained. Hardware results demonstrate that preprocessing can easily be performed during the acquisition phase, and multimodal biometric recognition can be treated almost instantly (0.4 ms on FPGA). We show the feasibility of a robust and efficient multimodal hardware biometric system that offers several advantages, such as user-friendliness and flexibility. (C) 2011 Society of Photo-Optical Instrumentation Engineers. [DOI: 10.1117/1.3534199]
\end{abstract}

Subject terms: multimodal biometrics; face recognition; contactless palmprint recognition; small-number sample sets; palm codes; score fusion; hardware implementation.

Paper 090932RRR received Nov. 24, 2009; revised manuscript received Nov. 16 2010; accepted for publication Dec. 7, 2010; published online Feb. 00, 2011.

\begin{abstract}
зо 1 Introduction
31 Biometrics has drawn extensive attention during the past 30 32 years for its huge potential in many applications, such as 33 building/store access control, suspect identification, surveil34 lance, and human computer interfacing. The key issue of 35 these applications is the identification of individuals by their 36 physiological or behavioral characteristics (e.g., face, finger37 print, iris, signature, or gait). Each biometric characteristic 38 has its own strengths and weaknesses: unimodal biometric systems have to contend with a variety of problems, such 40 as noisy data, nonuniversality, spoof attacks, and unaccept41 able error rates. In the past few years, researchers have more and more focused on the possibility of including multiple sources of information. Such systems, known as multimodal biometric systems, are more reliable. ${ }^{1}$

In many real-world applications, the number of available training samples is small, especially in the case of largescale biometric systems. Typically, for the face recognition problem in identity documents, the number of images from each class is considerably limited: only one or two faces can be acquired from each person. Moreover, systems using less training samples have a shorter enrollment stage and are more pleasant for users. A small number sam53 ple sizes allows us to use little memory. Nevertheless, in 54 the small-number sample context, many statistical methods
\end{abstract}

0091-3286/2011/\$25.00 @ 2011 SPIE show poor generalization ability and degrade the classification performance. ${ }^{2}$ In this paper, a reliable and contactless general-public multimodal biometric system is presented. It respects the small-number sample constraint and tries to be user-friendly.

Palmprint can be used as a reliable human identifier because the pattern of ridges is unique and their details are permanent. Compared to other physical biometric characteristics, palmprint biometrics have several advantages: lowintrusiveness, stable line features, and low-cost capturing device. ${ }^{3}$ Although palmprint is traditionally a contacting biometric, we use it without contact, which allows us to keep a pleasant and hygienic system. For that matter, an increasing number of works have interest in the use of contactless sensors. $^{3-5}$

Face is one of the most studied and commercialized biometrics. It is well accepted because humans routinely use facial information to recognize each other. But it suffers from some weaknesses: it is particularly affected by pose, expression, or illumination. In the past decades, a lot of face recognition algorithms have been proposed: statistical analysis as principal component analysis (PCA), independent component analysis (ICA), or linear discriminant analysis (LDA) ${ }^{6}$ neural networks $;^{7}$ graph matching; ${ }^{8}$ etc.

Fusion of face and palmprint is studied because it allows are to greatly improve performance while keeping a user-friendly and well-accepted system. Kumar and Zhang ${ }^{9}$ proposed a personal verification method combining 
83 palmprint, face, and claimed user identity to increase

84 authentication performance: a feed-forward neural network

85 is used to integrate individual matching scores and generate a

${ }_{86}$ combined decision score. Jing et al. ${ }^{6}$ use face and palmprint

87 for small-number sample recognition: the fusion occurred

88 at the pixel level on feature images is obtained due to

89 a Gabor filter bank. Zhang et al. ${ }^{10}$ present a geometry

90 preserving projection (GPP) approach to preserve the

91 interactions between the different modalities during the

92 subspace selection procedure: with GPP, all raw biometric

93 data (face, palmprint obtained with contact, and gait) from

94 the different identities and modalities are projected onto a

${ }_{5}$ unified subspace, on which classification is performed.

However, none of those methods are adapted to the calculation cost or memory constraints of embedded systems. Biometric algorithms work on raw and uncompressed images, whose processing requires a large number of operations. However, most of these operations are independent and can be performed on different parts of the image at 12 the same time. Because of this possibility of reaching a ${ }_{3}$ high parallelism degree, biometric algorithms are the right candidates for hardware implementation. For example, some research has been conducted in order to reduce the calculation time of monomodal biometric systems: Yang and Paindavoine ${ }^{11}$ have implemented a face-detection and recognition algorithm-based on radial basis function (RBF) neural network - on field-programmable gate array (FPGA), digital signal processor (DSP), and zero instruction set computer (ZISC) chips in order to compare the execution 112 times. Lopez-Ongil et al. ${ }^{12}$ present the FPGA implementa113 tion of an authentication system based on hand geometry, 114 which uses the continuous hamming distance to compare 115 hand dimension vectors. Other works explore multimodal 116 biometrics: Yoo et al. ${ }^{13}$ have developed two DSP systems 117 for iris-fingerprint and face-fingerprint recognition. In their 118 system, the most consuming tasks are implemented on 119 FGPA in order to increase the system speed.

120 The aim of our project is to build a reliable general-public 1 biometric system, that respects multiple constraints: hy22 gienic, low-cost, straightforwardness, user-friendliness, real123 time processing, limited memory, small sample set, etc. The 124 developed system could be used in businesses, hospitals, or 5 schools to control door opening, record hours worked by em126 ployees, restrict access to sensitive areas, control access to 127 school canteens, etc. Therefore, we present the hardware ar128 chitecture of a multimodal biometric recognition system with 129 massive exploitation of the inherent parallelism. Implemen30 tations are simulated on a Texas Instrument Digital Signal 1 Processor (DSP) and Xilinx Field Programmable Gate Array 2 (FPGA) platforms. DSPs are widespread processors that are 3 optimized to signal processing, whereas FPGAs are inex134 pensive devices adapted to parallel calculation that give the ability to quickly create a rapid and fully functional prototype that can emulate and verify solutions or even be embedded 7 into the final system. That is why we chose to implement our algorithm on these two devices. The remainder of the paper is organized as follows: Section 2 provides details of 140 the algorithm model from image acquisition to the steps of 141 fusion and decision, while Sec. 3 presents designed architec142 tures and their hardware implementations. Performance of 143 the system is presented in Sec. 4 and discussed in Sec. 5. 144 This is followed by the conclusion and presentation of the 145 perspectives in Sec. 6.

\section{Algorithm Model}

This section introduces the complete face and hand processing chain, which includes four principal steps: acquisition of images, hand preprocessing, palmprint and face feature extraction, and score fusion. A brief algorithm-oriented presentation of all the modules is available in Ref. 14.

\subsection{Acquisition of Images}

Traditional hand-based biometrics use contact with a surface ${ }_{153}$ and sometimes rigid placement guides. These have the ad- ${ }_{154}$ vantage of having a fixed focal field, and if they use pegs, 155 can rely on a standard placement. On the contrary, face is 156 a typical contactless biometric. We have designed a user- ${ }_{157}$ friendly system to acquire real-time hand and face images 158 that is totally contactless. Two low-cost Logitech QuickCam 159 Pro 9000 USB cameras are used with a maximum resolution $\quad 160$ of $1600 \times 1200$ to capture images under typical office lighting $\quad 161$ and daylight conditions.

Subjects enroll themselves thanks to an easily usable soft- ${ }_{163}$ ware. For the hand, they are only asked to place it horizontally $\quad 164$ and ensure that their fingers do not touch each other. Each ${ }_{165}$ subject could place his hand anywhere from a few dozen ${ }_{166}$ inches to a few inches from the sensor: the upper limit is ${ }_{167}$ defined by the position of a green background [see Fig. 1(a)]. ${ }_{168}$ Subjects must furthermore place their face in an enclosing $\quad 169$ frame of $360 \times 480$ pixels drawn on the webcam preview [see $\quad 170$ Fig. 1(b)]. Expression, accessories, and background are not 171 controlled: expression can vary from neutral to broad grin, ${ }_{172}$ and subjects choose to wear their eyeglasses or not.

\subsection{Image Preprocessing}

Working on palmprint in a contactless context requires some $\quad 175$ preprocessing. The region of interest (ROI) must indeed be 176 extracted from the hand image. Palm extraction requires hand 177 localization, followed by palm localization in the hand, and ${ }_{178}$

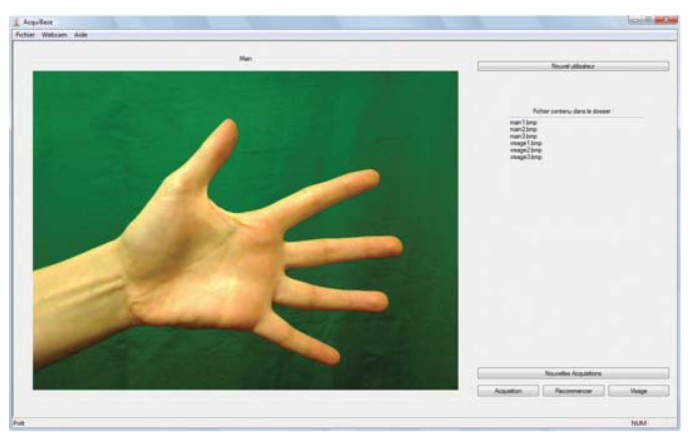

(a)

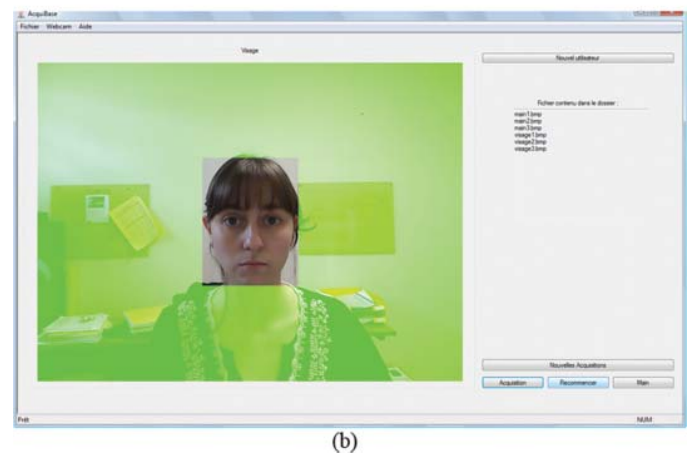

Fig. 1 Acquisition software: (a) palm interface and (b) face interface.

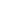

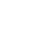

然

(1)

.
60 61 . . . .

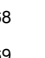
, 72 , 178 


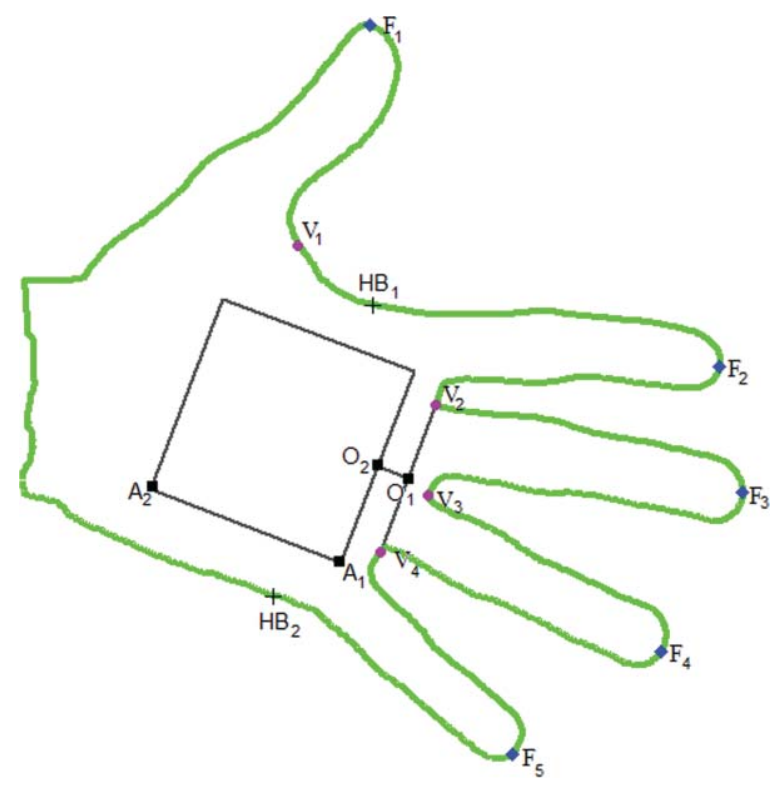

Fig. 2 Palm window definition.

179 then normalization because of the rotation and scale variation ${ }_{80}$ induced by the free placement. Hand segmentation consists ${ }_{11}$ of a thresholding on the red component of the RGB space: 182 because a green background has been chosen, the redder ${ }_{33}$ pixels belong to the hand. Some morphological operations 134 are also used in order to enhance the hand edges. After this 185 step, multiple reference points are defined; they correspond to 186 the fingertips and valleys between fingers. This localization ${ }_{187}$ of the hand extremities is achieved in two steps.

First, a contour extraction is performed using an eightneighborhood-borders tracking algorithm known as the Freeman algorithm. Second, hand extremities's locations are found. As subject fingers are located on the right of the image, local minima and maxima of the hand contour abscissa can be considered as fingertips and valleys. Because these initialized locations are not accurate, we applied a refining algorithm inspired by the method described in Ref. 5, which minimizes the euclidean distance between the considered point and its two neighbors among the reference points.

Doublet et al. ${ }^{4}$ propose a simple and efficient method to extract the palm from the location of such characteristic points. Our adaptation of this process consists of two steps: First, adding two characteristic points in order to calculate the hand width, and second localizing the palm window corners. Location of the new fiducial points is deduced from the length of the index and little fingers. Figure 2 shows the square window, which corresponds to the ROI. The distances $\left\|O_{1} O_{2}\right\|$ and $\left\|A_{1} A_{2}\right\|$ depend on the distance between the hand and the camera. Therefore, they are taken proportional to the hand width $\left(\left\|H B_{1} H B_{2}\right\|\right)$.

Because the palmprint images are of different sizes and orientations, we normalize them. First, they are rotated around the vertical axis. Then, they are resized to a standard image size of $64 \times 64$ pixels and converted into a gray-level image.

Because of the experimental setup, the pose of the face varies only slightly. Moreover, as we work on low-resolution images, it is not necessary to extract ROI. That is why the face preprocessing only takes up the last palm preprocessing steps: resizing to $64 \times 64$ pixels and conversion into a gray- 218 level image.

\subsection{Gabor Feature Extraction}

Palmprints exhibit a rich pattern of striations that enable dis- 221 criminating between people. Therefore, most of the studies 222 in palmprint recognition treat palmprints as textured images 223 and apply well-known pattern recognition techniques, such 224 as wavelets, ${ }^{15}$ PCA or ICA, ${ }^{16}$ and many others. Because of ${ }_{225}$ its good performance and specific qualities of luminosity ro- 226 bustness and frequency location, the Gabor filter is the most 227 efficient and popular tool. ${ }^{1,6,17}$

Face recognition is a mature biometric for which many 229 recognition approaches exist. Nevertheless, classical meth- 230 ods such as Eigenface or Fisherface are not adapted to the 231 small sample set problem, as explained in Ref. 2 or $18 . \quad 232$ Therefore, many variants of these algorithms have been 233 proposed in order to improve recognition performance in 234 this situation. ${ }^{19,20}$ Other methods, which combine image fil- 235 tering by a Gabor filter bank and PCA (Ref. 6) or LDA ${ }_{236}$ (Ref. 21) have also been studied to solve the small-number ${ }_{237}$ sample set problem. However, all these methods based on 238 statistical analysis require too high calculation complexity 239 and too much memory to be used in embedded systems. 240 However, some studies look into the use of one or more per- 241 tinent Gabor filters, ${ }^{22,23}$ which is the same principle as our 242 palmprint recognition algorithm.

Here, this filter is used to extract palmprint and face fea- 244 tures: a coding-based method is employed, that is founded 245 on the works of Refs. 4 and 24. This choice is also con- 246 sistent with the electronic embedded system context: regular 247 calculations, such as convolution operation, are easily imple- 248 mented on hardware systems and reduce power consumption. 249 Moreover, applying the same method on both palmprint and 250 face will facilitate hardware implementations.

A variety of implementations of this filter exists. Considering its performance and the need to reduce computation ${ }_{253}$ time and memory consumption, we use the ellipsoidal filter 254 in the real domain proposed in Ref. 4,

$G(x, y)=\exp \left[-\frac{x^{\prime 2}+\gamma^{2} y^{\prime 2}}{2 \sigma^{2}}\right] \cos \left(2 \pi \frac{0.56 x^{\prime}}{\sigma}\right)$,

where

$\left\{\begin{array}{l}x^{\prime}=\left(x-x_{0}\right) \cos (\Theta)-\left(y-y_{0}\right) \sin (\Theta) \\ y^{\prime}=\left(x-x_{0}\right) \sin (\Theta)+\left(y-y_{0}\right) \cos (\Theta)\end{array}\right.$.

The couple $\left(x_{0}, y_{0}\right)$ defines the function center, $\Theta$ controls the ${ }_{257}$ orientation, $\sigma$ is the standard deviation of the Gaussian factor, 258 and $\gamma$ is the spatial aspect ratio of this ellipsoidal function 259 fixed at 0.5 . For more luminosity robustness, the filter is 260 normalized by the subtraction of the coefficient average from 261 each coefficient.

Gabor palmprint features are obtained by the convolution 263 of the image with a single Gabor filter (whose coefficients 264 are empirically chosen, see Sec. 5), followed by a threshold- 265 ing operation with a threshold equal to 0 . This binarization 266 limits the characteristic size and the computation time in the 267 comparison phase. The feature extraction step is illustrated 268 in Fig. 3. For identity classification and verification, a sim- 269 ilarity measurement must be created in order to compare 270 the extracted parameters. For this matching process, we use 271 the traditional comparison method of binary matrices: the 272 


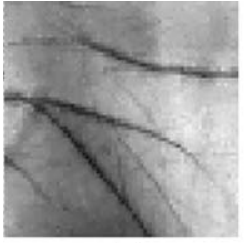

(a)

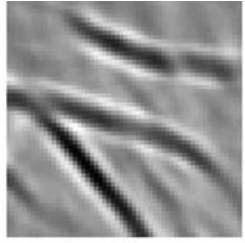

(b)

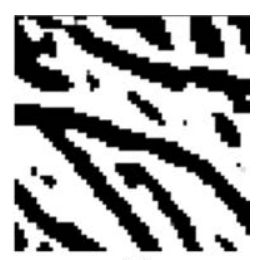

(c)
Fig. 3 Feature extraction of the palm: (a) corresponding images, (b) gabor features $(\Theta=\pi / 4, \sigma=4.6)$, and (c) final feature matrix.

273 Hamming distance, which is a pixel-by-pixel comparison 274 using the Boolean operator $\oplus$ (XOR).

Because palmprint and face localizations are not necessar276 ily ideal, we introduce a tolerance in translation by calculat277 ing the distance for multiple shifts and taking the minimum. 278 The final matching measurement for two feature matrices $A$ 279 and $B$ of size $N \times N$ is

$$
\begin{aligned}
& D(A, B) \\
& =\min _{|x|,|y|<2}\left[\sum_{i=0}^{N} \sum_{j=0}^{N} T\{A(i, j), x, y\} \oplus B(i, j)\right],
\end{aligned}
$$

280 where $T\{A, x, y\}$ is the translation of image $A$ horizontally 281 by $x$ and vertically by $y$.

\subsection{Fusion Scheme}

${ }_{283}$ Combining one or more biometric traits provides new in284 dependent information that gives the opportunity to greatly 285 improve recognition performance. Furthermore, it increases 286 the probability that one of the traits suits the user, which gives 287 a larger population coverage and complicates spoof attacks 288 by requiring more kinds of information.

289 A generic biometric system includes four principal steps: 290 data acquisition, feature extraction, matching to the template 291 database, and decision. Information fusion can occur at any of 292 the aforementioned steps. Most studies agree on the fact that 293 integrating information at an early stage of processing is more 294 effective than performing integration at a later stage. ${ }^{1}$ Earlier 295 stages contain richer information about the input biometric 296 data than later stages. However, fusing pixels or feature vectors implies a high compatibility between fused data and 297 does not allow modality-adapted processing, as in our case. 298

We use fusion at score level because there is sufficient 299 information content at this step and it is easy to access 300 and combine the matching scores. Savic and Pavesic ${ }^{25}$ have 301 demonstrated that the combination approach performs bet- 302 ter in biometric systems. Therefore, tree combination rules 303 have been tested. Let $P_{i}$ be the score obtained thanks to the ${ }_{304}$ matching between the current palmprint feature and the $i$ th 305 template of the palmprint matching base, let $F_{i}$ be the score 306 obtained thanks to the matching between the current face fea- 307 ture and the $i$ th face template, the corresponding final score 308 Fus $_{i}$ can be calculated from the minimum [Eq. (4)], the sum 309 [Eq. (5)], and the multiplication Eq. (6) rules as follows: 310

$\operatorname{Fus}_{i}=\min \left(P_{i}, F_{i}\right)$,

$\operatorname{Fus}_{i}=P_{i}+F_{i}$,

$\mathrm{Fus}_{i}=P_{i} \times F_{i}$

The final decision of the classifier is then given by choos- 313 ing the class that minimizes the fused matching measures 314 between the sample and all templates of the matching base. 315

If at least one of the two scores is low enough to suc- 316 cess in the recognition task, the fused score (obtained by 317 minimum, sum, or multiplication rules) would also allow 318 one to succeed in this task. That is why multimodal systems 319 outperform unimodal systems and increase the population 320 coverage: If one modality is vulnerable to certain condi- 321 tions, then the others take over. The way we designed the ${ }_{322}$ system (see Fig. 4) allows us, moreover, to use palmprint ${ }_{323}$ only, face only, or fusion of the two. Using this architecture 324 makes it possible to add other textured modalities, such as 325 knuckleprint or ear.

\section{Hardware Implementations}

327

Each of the proposed algorithms respects the embedded sys- 328 tem constraints. They work in particular with a low calcu- ${ }_{329}$ lation cost and low memory, which makes them particu- ${ }_{330}$ larly suitable for DSP implementation. Moreover the coding 331 scheme proposes a high potential of parallelization, which ${ }_{332}$ could be fully exploited by application-specific integrated

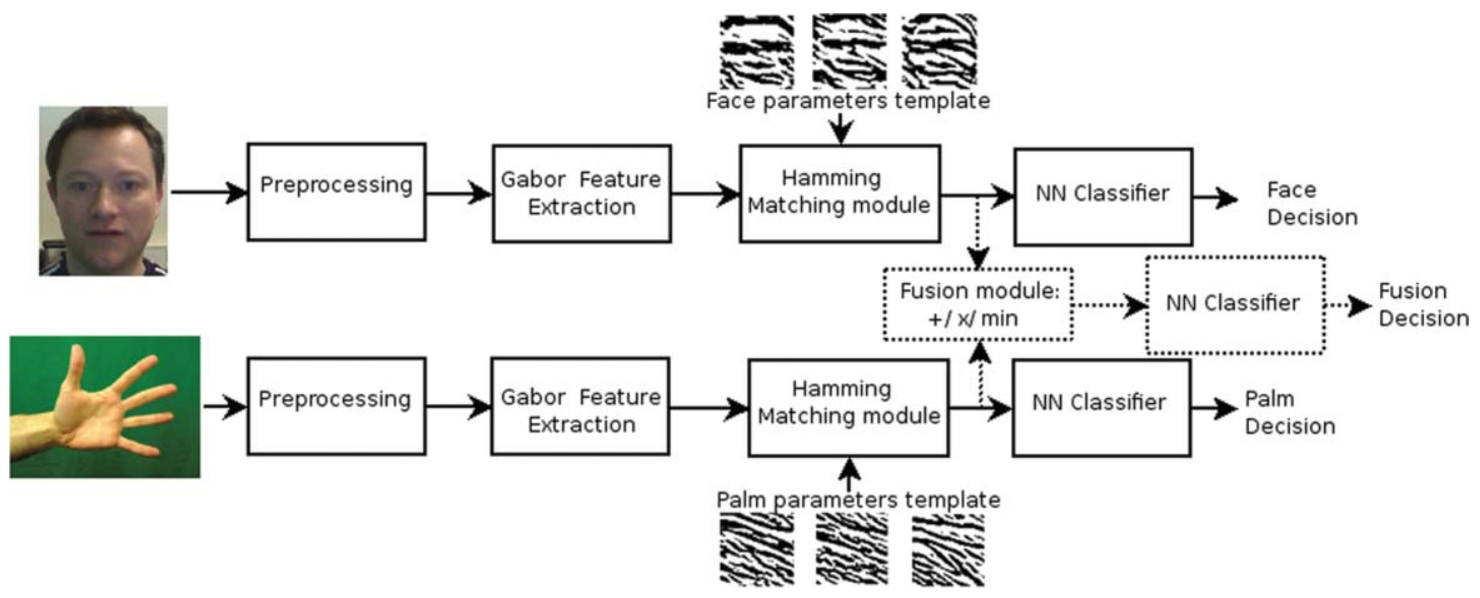

Fig. 4 Entire processing chain with possible score fusion using nearest-neighbor (NN) classifier. 


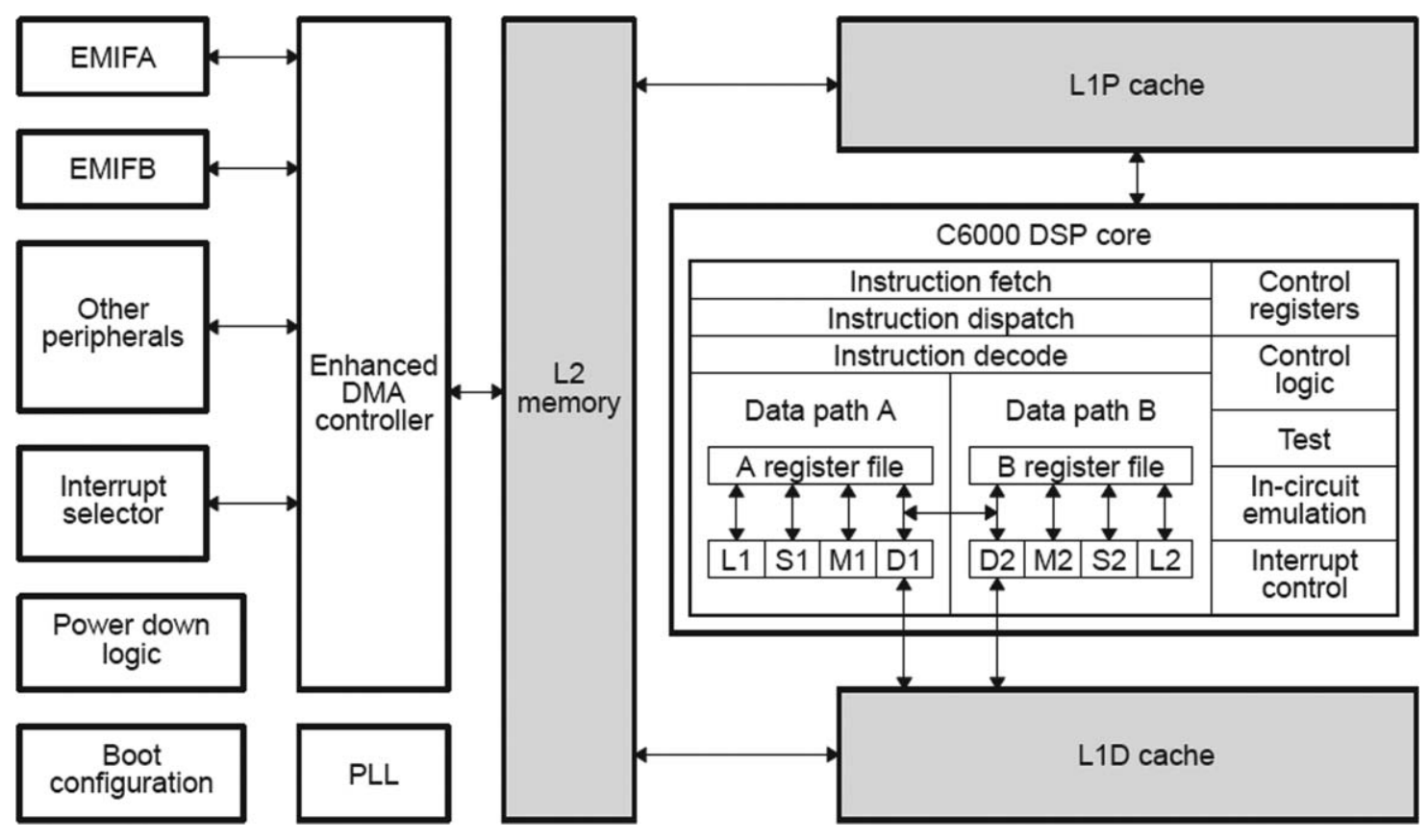

Fig. 5 TMS320C64x DSP block diagram.

334 circuit (ASIC) or FPGA. We propose the implementation of 335 the entire system on a Texas Instrument DSP platform, and 336 the implementation of the multimodal recognition step on a 337 Xilinx FPGA platform. Implementing the proposed multi338 modal recognition chain in FPGA efficiently is the key step 339 of an ASIC solution design.

\subsection{DSP Implementation}

The implementation of the processing chain has been simulated on a TMS320C64xx DSP platform of Texas Instruments ${ }^{26}$ thanks to the Code Composer Studio (CCStudio) tool. Such platforms are particularly well adapted to classical image processing algorithms, and allow one, at the same time, to easily implement more sophisticated processing. The C64x central processing unit (CPU), as shown in Fig. 5, consists of eight functional units, two register files, and two data paths. Devices of the c64x family can execute, for example, four 16-bit $\times 16$-bit multiplies every cycle, or eight 8-bit $\times 8$-bit multiplies. They have a two-level memory architecture for program and data. The first-level program cache is designated L1P on Fig. 5, and the first-level data cache is designated L1D. Both the program and data memory share the second-level memory, designated as L2, which is configurable and can provide up to $1024 \mathrm{~KB}$ of on-chip SRAM. A DSP implementation description has been made in C language. After an optimization step, we let the compiler of the CCStudio environment decide the possibilities of parallelization.

The number of CPU cycles required for the palmprint extraction depends of the hand shape. Our simulations empirically show that it is between $350 \times 10^{6}$ and $390 \times 10^{6}$, which corresponds to 350 and $390 \mathrm{~ms}$ at a frequency of $1 \mathrm{GHz}$. With such a short execution time, palmprint extraction can be performed during face image acquisition. The face preprocessing always uses the same number of CPU cycles, which is lower than $6 \times 10^{6}$ and corresponds to an execution time of $6 \mathrm{~ms}$. Face preprocessing could run in real time while 369 storing pixels. For these first hardware implementations, we 370 have chosen to work on a database of 25 people with two 371 samples per individual in the matching base. Guided by the 372 results of our algorithmic model (see Sec. 4 or Ref. 14), we 373 have chosen to perform the fusion thanks to the sum rule. 374 As feature samples are $52 \times 52$ binary matrices, the total size 375 of the base is only of $16 \mathrm{~KB}$. The coding scheme and the 376 recognition step requires about $7 \times 10^{6} \mathrm{CPU}$ cycles, which 377 corresponds to $7 \mathrm{~ms}$.

Although parallelization possibilities are high for this kind 379 of device, parallelism potential of the face and palmprint 380 recognition algorithms is only lightly exploited on a DSP. ${ }_{381}$ That is why, we have also simulated the hardware imple- 382 mentation of the last steps of the processing chain (fea- ${ }_{383}$ ture extraction, matching, fusion, and decision) on an FPGA 384 platform.

\subsection{FPGA Implementation}

386

We work on a Virtex-5-XC5VFX70T FPGA of the Xilinx 387 society. ${ }^{27}$ It has been chosen for its configuration: It con- ${ }_{388}$ tains, in particular, 128 DSP slices (with $25 \times 18$ multipli- ${ }_{389}$ ers and 48-bit adder/subtracter/accumulator), which support 390 massively parallel digital signal processing algorithms, and 391 22,400 configurable logic blocks (CLBs). Slices of the CLBs 392 can be used to provide logic, arithmetic, and ROM functions; 393 a part of them can also be used as distributing RAM or 32-bit 394 data registers.

FPGA implementations have been simulated with the Very ${ }_{396}$ High-Speed Integrated Circuit, Hardware Description Lan- ${ }_{397}$ guage (VHDL) description using the Xilinx ISE tool. Results 398 of the FPGA implementations will be presented in terms of 399 used resources and processing speed. As for the DSP imple- 400 mentation, we have worked on a database of 25 people with 401 two samples per individual in the matching base and we use 402 the sum rule. 


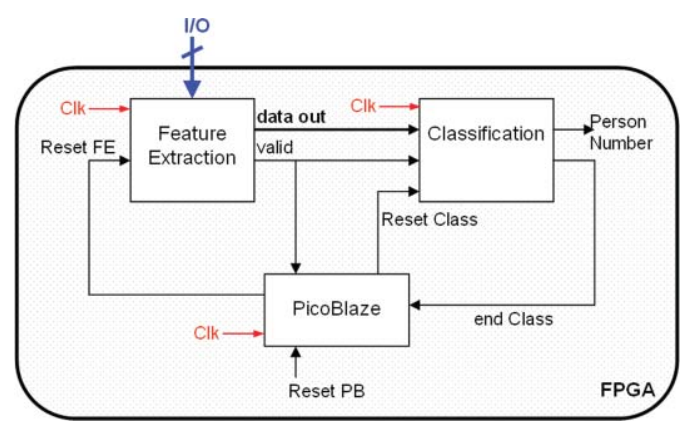

Fig. 6 Recognition chain hardware realization on FPGA: the software microcontroller core PicoBlaze (PB) controls the feature extraction (FE) and classification (Class) blocks.

$404 \quad$ Figure 6 displays the entire recognition chain. We use 405 a PicoBlaze (PB) microcontroller core implemented on the 406 FPGA in order to synchronize the two stages of the palm407 print recognition chain [i.e., the feature extraction (FE) and 408 the classification (Class)]. PB is intellectual property of the 409 ISE software, ${ }^{27}$ this softcore microcontroller is programmed 410 in assembly. It triggers the FE block when a palmprint image 411 arrives in the FPGA, triggers it again when a face image ar412 rives, and starts the Class block when the FE block processing ${ }_{413}$ is finished. When the Class block provides the template num414 ber, which corresponds to the person's identity, the complete 415 system is ready for the next recognition.

${ }_{416}$ Figure 7 displays the proposed design of the feature ex417 traction block. We can see that data parallelism is fully

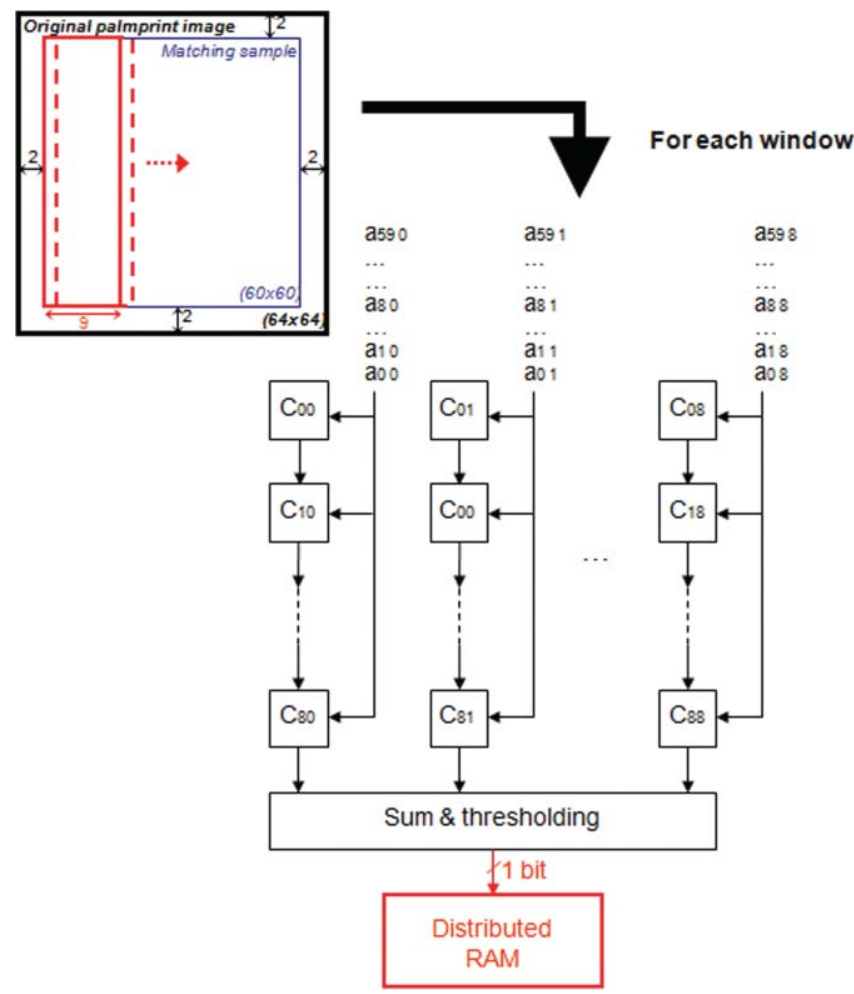

Fig. 7 Parallel structure for feature extraction stage: (a) the palmprint image is distributed in successive windows of $60 \times 9$ pixels, (b) feature extraction is realized using an architecture composed of 9 lines $\times 9$ columns of DSP slices that perform operations simultaneously.

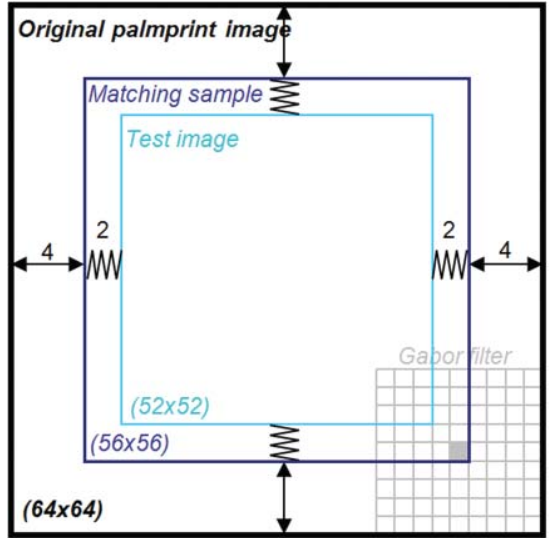

Fig. 8 Image sizes during processing: original images $=64 \times 64$ pixels, training sample images $=56 \times 56$ pixels because of the convolution with a $9 \times 9$ Gabor filter, and test images $=52 \times 52$ pixels because of the $2 \times 2$ pixels margin introduced by the elastic matching.

exploited using the pipeline technique. In agreement with 418 Fig. 8, each final test image size is $52 \times 52$ pixels. Because ${ }_{419}$ original images are larger than needed, border pixels are 420 not used and we work on the $60 \times 60$ central pixels. In this 421 module, the original image is stored in a Block RAM and ${ }_{422}$ processed by windows of $60 \times 9$ pixels. The process ends 423 after 52 shifts of the vertical window. The convolution op- 424 eration is realized using a structure of 81 DSP slices. Each 425 of these slices multiplies a received pixel value with a filter ${ }_{426}$ coefficient and accumulates the previous result.

A total of $61 \times 52=3172$ clock cycles are necessary in order to run this feature extraction block; 89 DSP slices, ${ }_{429}$ 1 Block RAM and 187 slices are used. The corresponding 430 operating frequency is equal to $175 \mathrm{MHz}$.

The classification module consists of the calculation of ${ }_{432}$ 100 elastic Hamming distances $\left(25\right.$ templates $\times 2$ samples ${ }_{433}$ $\times 2$ biometrics), followed by 50 score fusions (each palm ${ }_{434}$ score is fused with the corresponding face score), and a ${ }_{435}$ comparison between each of these fused scores (NN clas- ${ }_{436}$ sification). The 100 templates have been performed off- ${ }_{437}$ line and loaded in distributed RAM during the hardware- 438 configuration phase. Moreover, each elastic Hamming 439 distance is performed by the calculation of 25 Hamming 440 distances.

Figure 9 illustrates hardware realization of the elastic ${ }_{442}$ matching stage. We have chosen to carry out this step in 25443 iterations corresponding to the 25 shifts of the elastic dis- 444 tance. We have designed a logic block in order to per- ${ }_{445}$ form horizontal and vertical shift control. At each iter- 446 ation, 100 Hamming distances are calculated in parallel. 447 An inner loop provides in parallel 100 XOR operation re- 448 sults to 100 accumulators. When this loop is completed af- ${ }_{449}$ ter $2704(=52 \times 52)$ cycles, each accumulator provides a 450 Hamming distance value, which can be compared to prece- 451 dent values. The 100 minima are stored in registers of the ${ }_{452}$ FPGA. At the end of the 25 iterations, the fusion occurs ${ }_{453}$ by summing scores two by two. A final comparison step 454 then finds the minimal value among the 50 fused min- 455 ima. The person's identity is given by the corresponding ${ }_{456}$ template number. A total of $(2704+1) \times 25($ XOR $)+1($ sum $) \quad 457$ +55 (final comparison $)=67681$ clock cycles are necessary 458 in order to perform this elastic matching stage. 


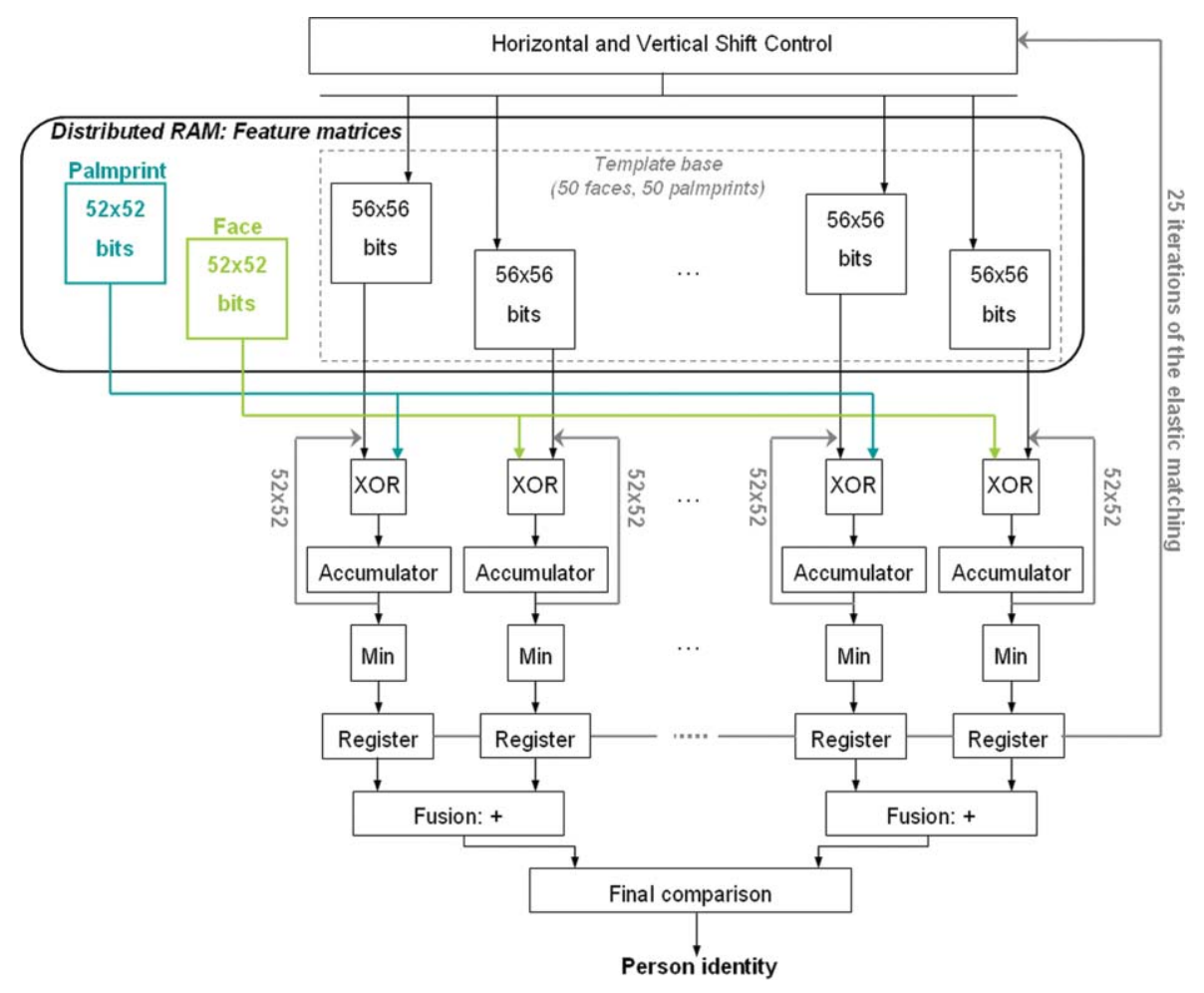

Fig. 9 Implementation of the classification block: for each of the 25 iterations, 100 Hamming distances are performed in parallel.

The elastic matching step does not use DSP slice or Block RAM but only CLB resources: a total of 8035 slices are used. The obtained operating frequency is equal to $175 \mathrm{MHz}$.

The general operating frequency is equal to $175 \mathrm{MHz}$, it corresponds to the frequency of both EM and Class modules. Thus, because our processing needs about $3172 \times 2+67681$ clock cycles, the entire operating time is on the order of $423 \mu \mathrm{s}$.

Chosen algorithms respect the constraints of simplicity, low-cost, regularity, and low-memory use. Thanks to the parallelization work, the entire processing is performed in only $0.4 \mathrm{~ms}$. Moreover, implementations have been achieved using only a portion of the available resources of the Virtex5-XC5VFX70T FPGA (see Table 1). In particular, we use very few logical resources (total ratio of 19.1\%): because the Class block does not use DSP slice but only register slices and LUT slices, the number of recognizable people could be increased and reach 100.

Table 1 Hardware implementation results of the recognition chain on a Virtex-XC5VFX70T FPGA.

\begin{tabular}{cccc}
\hline Logic element & $\begin{array}{c}\text { Used } \\
\text { number }\end{array}$ & $\begin{array}{c}\text { Total } \\
\text { number }\end{array}$ & $\begin{array}{c}\text { Used ratio } \\
(\%)\end{array}$ \\
\hline DSP48 slices & 89 & 128 & 69.5 \\
Block RAMs & 2 & 148 & 1.4 \\
Slices & 8566 & 44800 & 19.1 \\
\hline \hline
\end{tabular}

\section{Extensive Experimental Results}

\subsection{Presentation of Experiments}

For this feasibility study, we built a database called the uB 480 (University of Burgundy) database. It consists of images from 481 130 people, with nine face images and nine hand images 482 per person. Pairs of images were recorded in three sessions 483 of three images. The period of time between each session 484 is spread from one day to a few weeks in order to take 485 into account luminosity variation and possible variation in 486 positioning or appearance. The acquisition environment is ${ }_{487}$ totally contactless and very user friendly (see Sec. 2.1). 488

In order to verify our approach, we also tested the process- 489 ing on a multimodal database, which consists in the fusion of 490 two public databases: the Hong Kong Polytechnic University 491 (PolyU) palmprint database ${ }^{28}$ and the AR face database. ${ }^{29}{ }_{492}$ The PolyU palmprint database contains 7752 gray-scale im- 493 ages from 386 different palms. Twenty samples from each of 494 these palms were collected in two sessions (of 10 samples). 495 The average interval between the first and second collec- 496 tion was two months. The size of every original image is 497 $384 \times 284$ pixels. Fig. 10 shows some original palm images 498 of the PolyU database. They have been obtained with contact 499 and pegs in controlled lighting conditions. ${ }^{30} 500$

Our palm extraction method has been adapted to the pro- 501 cessing proposed in Ref. 30: the fixed focal has been taken 502
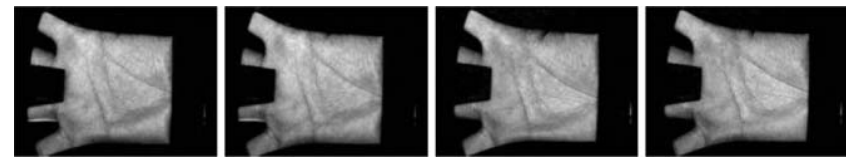

Fig. 10 Four images of the same palm from the PolyU database. 


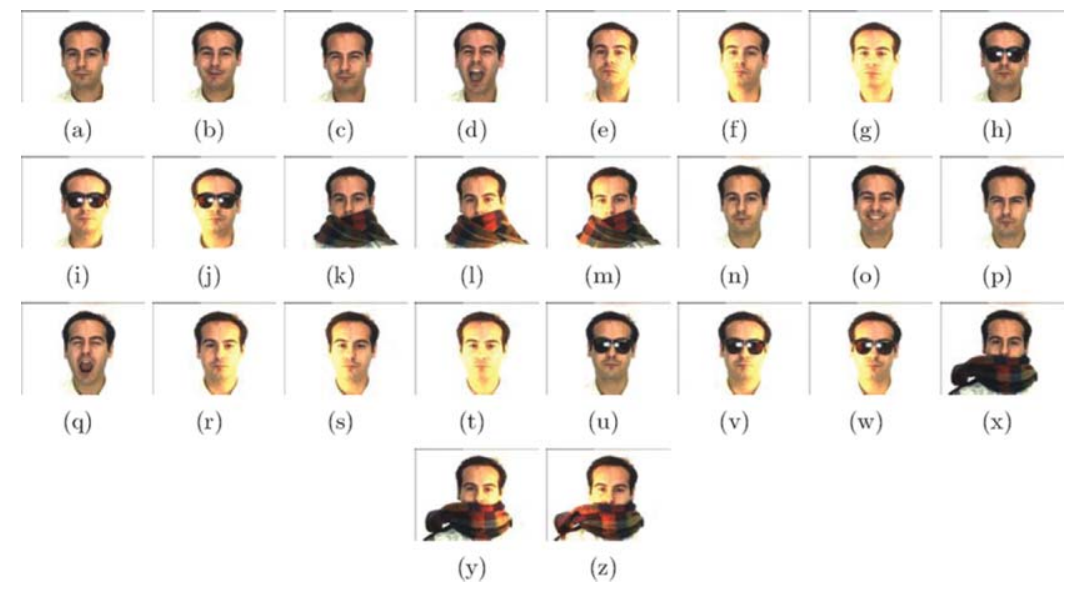

Fig. 11 Demonstration images of one subject from the AR database: $(a-m)$ are from Session 1 and $(n-z)$ are from session 2.

503 into account by using a fixed size window when defining 504 the ROI. Algorithms have also been adapted to the number 505 of visible fingers, which is no longer 5. Palmprints are still 506 rotated and scaled to the size of $64 \times 64$ pixels.

The AR face database is composed of $\sim 4000$ color face 508 images of 126 people (70 men and 56 women), including 509 frontal views of faces with different facial expressions, under 510 different lighting conditions, and with various occlusions ${ }^{29}$ 511 (see Fig. 11). Face images were acquired in two sessions 512 separated by two weeks. Each session captured 13 color 513 images. The two sessions are available for 119 individuals. 514 The preprocessing is the same as that of the uB database: 515 all color images are transformed into gray-level images and 516 each image (of $768 \times 576$ pixels) is scaled down to $64 \times 64$ 7 pixels.

518 We take sample subsets of the same size from these two 519 databases in order to create the multimodal database. As Jing ${ }_{520}$ et al., ${ }^{6}$ we use the first 119 palmprint classes with each class 521 containing all 20 samples and all 119 face classes with each 522 class including the first 20 samples.

The two Gabor filters have been chosen empirically on the ${ }_{524} \mathrm{uB}$ database and applied to both $\mathrm{uB}$ and AR-PolyU databases. 525 The way the Gabor filter coefficients have been chosen is ${ }_{526}$ explained in Ref. 14. Actually, the chosen filter is the same 527 for the two modalities: its coefficients are set as $\lambda=8.20$ and ${ }_{528} \Theta=2 \pi / 8$.

${ }_{529}$ In this paper, all the results take into account the con530 straints of the hardware implementation. The preprocessing 531 algorithms have been adapted to fixed-point calculation (for 532 their DSP implementation), and the remaining processing ${ }_{533}$ has been quantified (for their FPGA implementation). In this 534 way, results of the hardware system can be compared to those 535 of the algorithmic system presented in Ref. 14.

\section{4.2 Recognition Performance}

537 The uB database contains $130 \times 9=1170$ images of each 538 modality. For the recognition tests, it is divided in two parts: 539 the training sample set and test sample set. As we respect 540 the small sample set constraint, the number of samples per ${ }_{541}$ person in the matching base varies from 1 to 3 . We defined 542 two different protocols to conduct our experiments. Proto543 col 1: samples of the matching base are picked up randomly 544 among the nine available ones, and all the remaining samples 545 are used for tests. Protocol 2: samples of the matching base are picked up randomly among the three available ones of a 546 unique session, and only the samples of the two other sessions ${ }_{547}$ are used for tests. Thus, when the matching base contains 548 $n$ samples per person $(n \in\{1,2,3\}),(9-n) \times 130$ tests are 549 performed according to the protocol 1 and $6 \times 130(=780) \quad 550$ according to the protocol 2. Protocol 1 is the most used in 551 studies because it allows one to take into consideration all the 552 information contained in the database. Protocol 2 is used to 553 verify the robustness of the algorithm in more realistic condi- 554 tions: in the real world, all the matching samples are acquired 555 during the enrollment phase, so the captured variability is 556 reduced.

Results are qualified by the recognition rate, which is the 558 ratio between the number of correct classification results and 559 the total number of tests. Because it depends on the selected 560 samples, nine tests with nine different matching bases are 561 performed (for a matching base built according to protocol 2562 in the three samples cases, only three tests are performed, 563 since it is only possible to build three different bases). They 564 are then averaged to constitute a final result [the for aver- 565 aged recognition rate (ARR)], which objectively describes 566 the performance of the system.

Results obtained thanks to the protocol 1 are given in 568 Table 2. They are very similar to those of our former 569 algorithmic study: ${ }^{14}$ quantification of the Gabor filtering and 570 transition to fixed-point do not introduce any performance 571 degradation. As with the algorithmic model, the palmprint 572

Table 2 Average recognition rate obtained according to the protocol 1 on the $\mathrm{uB}$ database.

\begin{tabular}{lccc}
\hline \hline Method & $\begin{array}{c}\text { ARR (\%) } \\
\text { one sample }\end{array}$ & $\begin{array}{c}\text { ARR (\%) } \\
\text { two samples }\end{array}$ & $\begin{array}{c}\text { ARR (\%) } \\
\text { three samples }\end{array}$ \\
\hline Face recognition & $79.10 \pm 2.71$ & $90.93 \pm 4.60$ & $95.25 \pm 6.93$ \\
Palm recognition & $91.05 \pm 1.22$ & $96.82 \pm 1.52$ & $98.27 \pm 1.84$ \\
Minimum score & $92.43 \pm 1.70$ & $97.40 \pm 2.12$ & $98.79 \pm 2.63$ \\
Summed score & $96.02 \pm 0.95$ & $98.96 \pm 0.71$ & $99.59 \pm 0.76$ \\
Multiplied score & $96.38 \pm 0.94$ & $99.07 \pm 0.65$ & $99.61 \pm 0.79$ \\
\hline \hline
\end{tabular}


Table 3 Average recognition rate obtained according to the protocol 2 on uB the database.

\begin{tabular}{lccc}
\hline \hline Method & $\begin{array}{c}\text { ARR (\%) } \\
\text { one sample }\end{array}$ & $\begin{array}{c}\text { ARR (\%) } \\
\text { two samples }\end{array}$ & $\begin{array}{c}\text { ARR (\%) } \\
\text { three samples }\end{array}$ \\
\hline Face recognition & $73.50 \pm 2.35$ & $81.85 \pm 2.19$ & $84.96 \pm 2.52$ \\
Palm recognition & $89.32 \pm 1.54$ & $94.06 \pm 1.26$ & $95.56 \pm 0.85$ \\
Minimum score & $90.16 \pm 1.51$ & $93.69 \pm 1.04$ & $94.74 \pm 1.22$ \\
Summed score & $94.89 \pm 0.82$ & $97.68 \pm 0.66$ & $98.46 \pm 0.56$ \\
Multiplied score & $95.31 \pm 0.77$ & $97.89 \pm 0.62$ & $98.42 \pm 0.52$ \\
\hline \hline
\end{tabular}

573 recognition chain achieves, alone, a high-performance level. 574 Face recognition does not perform as well as palm recogni575 tion, but results are rather high for such a low-computational576 cost method in natural illumination conditions. Fusion 577 always performs better than unimodality, and the difference 578 between fusion methods is low: averaged recognition rates 579 differ only by a few tenths. Considering the computational 580 cost and the results of each method, the addition is very 581 interesting in our case. Minimum has a low complexity 582 but does not give good results, and the small performance 583 increase induced by the multiplication does not compensate 584 the difference of cost. There is a high similarity between the 585 sum and multiplication rules. Very good results are obtained 586 in the two-samples case: error is $\sim 1 \%$. It can be noted 587 that performance grows substantially between the one- and swo-sample cases, while the increase between the two89 and three-samples cases is minor.

Results obtained thanks to protocol 2 are presented in Table 3. As expected, they are generally not as good as those obtained according to protocol 1 . However, they are still high: ARR after fusion is between $94.9 \%$ in the one-sample case and $98.5 \%$ in the two-sample case. All the comments made for the Table 2 are applicable to Table 3: fusion allows one to 56 substantially increase the performance, there is only a small 59 difference between addition and multiplication, and the gap 598 between the one- and two-sample cases is significant. The
Table 4 Average recognition rate obtained with 20 random tests for each method using the AR-PolyU database.

\begin{tabular}{lccc}
\hline \hline Method & $\begin{array}{c}\text { ARR (\%) } \\
\text { one sample }\end{array}$ & $\begin{array}{c}\text { ARR (\%) } \\
\text { two samples }\end{array}$ & $\begin{array}{c}\text { ARR (\%) } \\
\text { three samples }\end{array}$ \\
\hline Face recognition & $68.22 \pm 3.36$ & $83.69 \pm 10.4$ & $86.21 \pm 9.97$ \\
Palm recognition & $85.46 \pm 1.29$ & $93.90 \pm 0.77$ & $96.03 \pm 0.58$ \\
Minimum score & $71.95 \pm 2.78$ & $86.15 \pm 9.66$ & $88.27 \pm 9.13$ \\
Summed score & $92.04 \pm 1.18$ & $97.49 \pm 0.95$ & $98.48 \pm 0.63$ \\
Multiplied score & $92.99 \pm 1.11$ & $97.92 \pm 1.70$ & $98.66 \pm 1.25$ \\
\hline \hline
\end{tabular}

only difference lies in the results of the minimum fusion, 599 which does not bring a performance increase to the palm- 600 print recognition. Moreover, we can see that fusion is more 601 robust than monomodality: when the variability captured in 602 the sample base decreases, the standard deviation of the face ${ }_{603}$ and palm results are greatly reduced, whereas it keeps similar 604 values for the fusion.

Table 4 illustrates the average results of 20 random tests conducted on AR-PolyU database according to the protocol 607 described in Ref. 6. We can see that all trends revealed by the 608 tests conducted on the $\mathrm{uB}$ database are confirmed on these 609 public databases.

For the face, errors are typically caused by the occasional 611 wear of accessories (such as glasses) and by changes in ex- 612 pression or pose. For the palm, they are often due to a lack ${ }_{613}$ of image quality (bad focus, inhomogeneous illumination, 614 etc.). These criteria are not correlated. That is why, most 615 of the time, only one modality fails when a pair of images 616 is tested. The fusion of the two often brings enough infor- 617 mation to override the confusion: for example, the sum of 618 two small distances (calculated on the samples of the same 619 user) can be smaller than the sum between a very small dis- 620 tance (calculated on the samples, which are confused) and a 621 large one (calculated on the samples of the other modality, 622 which are not confused). Sometimes, both modalities are mis- 623 taken, but the overall system succeeds, as in Fig. 12. This is 624

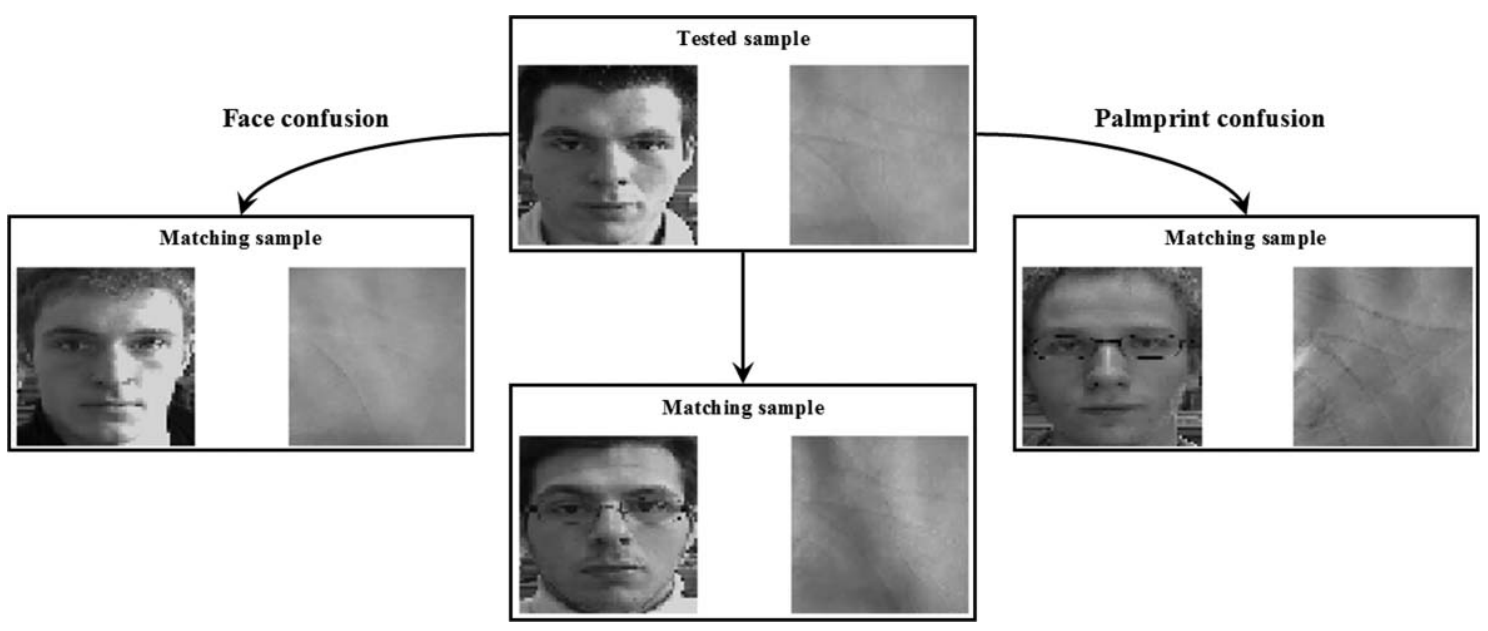

Fig. 12 Example of overall system success despite failure of the monomodal systems. 
Table 5 Small sample biometric recognition performance comparison using the AR face database and PolyU palmprint database. Average recognition rate in the two- and three-sample cases.

\begin{tabular}{|c|c|c|c|c|c|c|}
\hline \multirow[b]{2}{*}{ Method } & \multicolumn{3}{|c|}{$\begin{array}{c}\text { ARR (\%) } \\
\text { two-samples case }\end{array}$} & \multicolumn{3}{|c|}{$\begin{array}{c}\text { ARR }(\%) \\
\text { three-samples case }\end{array}$} \\
\hline & Face & Palm & Fusion & Face & Palm & Fusion \\
\hline Jing et al. & 65.67 & 63.33 & 92.66 & 74.88 & 64.29 & 96.14 \\
\hline Proposed method & 83.69 & 93.90 & 97.49 & 86.21 & 96.03 & 98.48 \\
\hline
\end{tabular}

${ }^{\text {a Reference } 6 .}$

625 probably because the two modalities are confused with sam626 ples of two different users, which cannot occur when they 627 are fused because they are considered simultaneously.

\section{4.3 Verification Performance}

629 Performance of biometric verification systems is measured 630 in terms of false rejection rate (FRR), which consists in 631 the error rate in the intraclass comparisons, and false ac632 ceptance rate (FAR), which is computed from the interclass 633 comparisons. A given FRR is achieved at a fixed FAR, and 634 vice versa. By varying the FRR (or the FAR), the receiver 635 operating characteristic (ROC) curve is obtained. In order 636 to judge the performance of a verification algorithm, it is 637 usual to use the operating point where the FAR and FRR 638 are equal. It corresponds to the so-called equal error rate 639 (EER).

640 In biometric verification systems, the test person is com641 pared to a single reference person and a decision is made 642 whether the two are identical or not. That is why biomet643 ric verification usually needs more images per individual for 644 training in order to capture intraclass variability. Therefore, 645 biometric verification often suffers more from the small sam646 ple size problem than biometric recognition. ${ }^{19}$

647 As for the biometric recognition, we arbitrarily take 648 1-3 samples of each of the 130 individuals in order to build 649 the training set. The remainder is used as test set. We com- pared each test sample to all training samples: for a given 650 test sample of the $\mathrm{uB}$ database, we perform genuine tests 651 with the samples of its own class, and impostor tests with the 652 samples of the other 129 classes. For example, in the three- 653 samples case, the system tests $780(130 \times 6)$ genuine users 654 and $100,620(130 \times 129 \times 6)$ impostors.

Table 6 gathers EERs calculated in the one-, two-, and 656 three-sample cases on the $\mathrm{uB}$ and AR-PolyU databases, and 657 Fig. 13 displays the ROC curves in the one-sample case. It has 658 to be noted that all results correspond to average verification 659 rates obtained by averaging the verifications rates of 9 or 660 20 random tests. We can see that verification follows the 661 same trends as recognition: palm achieves good performance 662 alone and fusion allows one to greatly improve the results. 663 Figure 13 shows that the curve behavior is the same on the 664 two multimodal databases and that fusion by addition and 665 multiplication is very similar.

\section{Discussion}

667

Proposed system not only reach good performance in terms 668 of hardware implementation, but also in terms of experi- 669 mental results: it obtains similar results to those we can 670 find in the literature. In the same conditions of biometric 671 recognition on the AR-PolyU database, Jing et al. ${ }^{6}$ obtain 672 slightly lower performance, which keeps the same trends 673 (see Table 5). For this, they use a Gabor feature 674

Table 6 Average equal error rate comparison for biometric verification. The bottom two rows correspond to the results obtained by Kumar et al. ${ }^{9}$ without using (A) and using (B) subject-claimed identity.

\begin{tabular}{lccccc}
\hline \hline & & & \multicolumn{3}{c}{ AEER (\%) } \\
\cline { 4 - 6 } Method & Database & Sample Size & Face & Palm & Fusion \\
\hline Proposed method & AR-PolyU & 1 & 15.1 & 10.9 & 6.21 \\
Proposed method & AR-PolyU & 2 & 7.07 & 4.62 & 2.38 \\
Proposed method & uB & 1 & 11.2 & 5.43 & 3.12 \\
Proposed method & uB & 2 & 5.22 & 2.23 & 1.10 \\
Proposed method & uB & 3 & 3.54 & 1.53 & 0.79 \\
Kumar et al. (A) & & 4 & 5.48 & 5.24 & 2.21 \\
Kumar et al. (B) & & 4 & 4.28 & 4.45 & 0.72 \\
\hline \hline
\end{tabular}

${ }^{\text {a Reference } 9 .}$ 


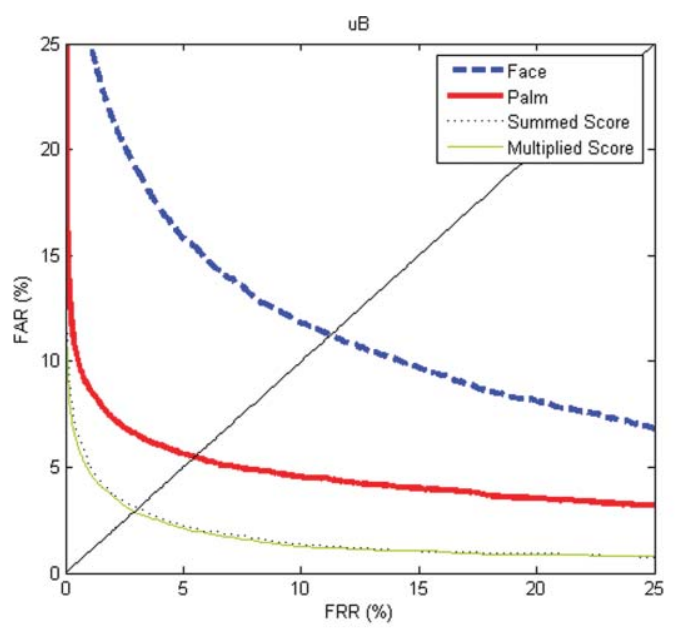

(a)

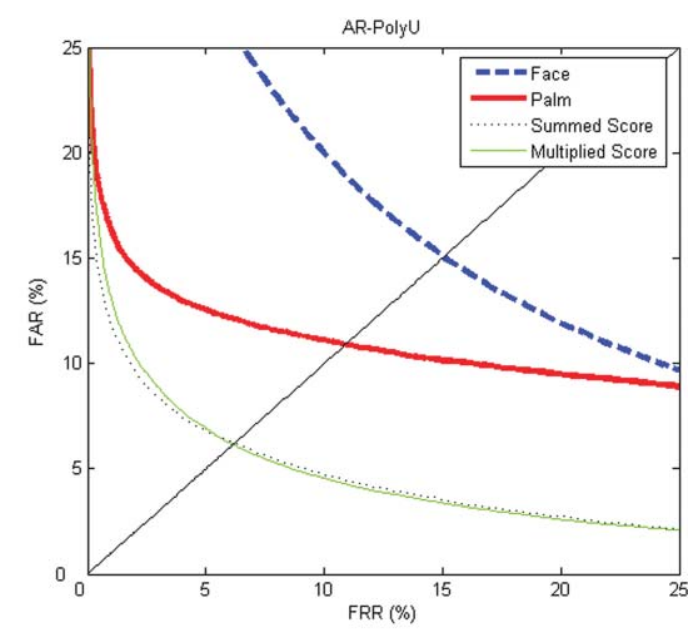

(b)

Fig. $13 \mathrm{ROC}$ curves of biometric verification in the one-sample case calculated on (a) the uB database and (b) the AR-PolyU multimodal database.

675 fusion followed by a feature compression using a Kernel Discriminative Common Vectors (KDCVs) approach 67 and a classification by the radial basis function (RBF) network.

Kumar et al. ${ }^{9}$ propose a score fusion using a feed-forward neural network trained on a base of four samples per person. Face features are extracted by the Eigenface method, and palmprint by the combination of four directional filters. The proposed method is tested on a multimodal database designed 84 by the authors that contains 70 subjects and is acquired in 5 more controlled conditions (for example, illumination, distance between hand and sensor). Table 6 tries to make some 67 biometric verification performance comparisons between the two bimodal systems. Kumar et al. ${ }^{9}$ show that combining subject claimed identity allows one to reduce verification error (EER from 2.21 to $0.72 \%$ ). On the uB database with only three samples, we also obtain better performance than this reference method, which does not use the claimed identity, and our EER is very similar to the one obtained using claimed subject identity. Results obtained with AR-PolyU in the twosample case are also comparable to those of the reference method.

It must be noted that performance calculated on the ARPolyU database is not as good as that calculated on the $u B$ database because hand images are of lower quality and do not show the entire hand, which makes palm extraction less accurate. Moreover, faces are not acquired in the same conditions and show a large white background.

In terms of hardware implementation, as Yang and Paindavoine $^{11}$ or Lopez-Ongil et al. ${ }^{12}$ (who work on face and hand geometry recognition, respectively), we prove that FPGA implementation is highly better than DSP (or general purpose processor) implementation. For Ref. 11 and 12 execution time is multiplied by 3 , and in our case it is multiplied by 17 . This high coefficient is achieved because we work on very short time $(0.4 \mathrm{~ms}$ for FPGA and $7 \mathrm{~ms}$ for DSP) and use massive parallelism on FPGA. If we compare the work of Yoo et al. ${ }^{13}$ on multimodal recognition to ours, then we can see that with comparable EER $(1.5 \%$ for the iris, for example), execution times are better (total execution time of $<500 \mathrm{~ms}$ for us and $\sim 1 \mathrm{~s}$ for the iris in Ref. 13).
We observe that with a sequential architecture the exe- 716 cution time of the last steps of processing depends on the 717 number of subjects in the comparison base. However, thanks 718 to the parallel architecture of the FPGA implementation, 719 recognition of 50 or more individuals could be realized using 720 the same chip (FPGA Virtex-XC5VFX70T) with the same 721 processing speed. On the other hand, authentication would 722 be even faster on DSP because the comparison base would 723 contain the samples of a single user.

\section{Conclusion and Perspectives}

725

In this paper, we have presented a contactless biometric sys- 726 tem that combines two modalities: palmprint and face. A ${ }_{727}$ complete processing chain has been developed from the ac- 728 quisition of hand and face images to classification decision, 729 and a hardware architecture has been implemented on DSP 730 and FPGA. Face and palmprint are two decorrelated modal- 731 ities, that can be acquired easily with minimal equipment 732 (a webcam) and without contact. Multimodal systems have ${ }_{733}$ many advantages over monomodal systems, such as better 734 robustness or greater universality. Therefore, using these two 735 biometrics in a multimodal system ensures one to create an 736 efficient general public system.

As we work on palmprint in a contactless context, a hand ${ }_{738}$ preprocessing (which consists of a palm extraction) has been $\quad 739$ developed and simulated on a DSP platform. Hardware im- 740 plementation of the rest of the multimodal recognition chain 741 has been simulated on the DSP and on a FPGA Virtex-5 de- 742 vice. Hardware results demonstrate that preprocessing can 743 easily be performed during the acquisition phase, and multi- 744 modal biometric recognition can be treated almost instantly. 745 Only $0.4 \mathrm{~ms}$ are necessary using 50 training samples recorded 746 on 25 persons with low-resource consumption on FPGA, 747 while no more than $7 \mathrm{~ms}$ are needed on DSP.

A database of 2340 images (130 subjects $\times 2$ modalities 749 $\times 9$ views) was built in real-world conditions (user-friendly 750 interface and natural illumination, for example). Experimen- 751 tal results show that multimodal fusion always reaches better 752 performance than monomodality. The proposed algorithm, 753 which is based on low-complexity operations, such as Gabor 754 filtering and similarity measurement by binary comparison, 755 
756 fits palmprint recognition particularly well. The fusion of 757 palmprint and face at score level allows us to achieve high 758 recognition rates $(98.96 \%$ using $\mathrm{uB}$ database and $97.49 \%$ 759 using AR-PolyU database with only two training samples 760 per person and per modality). In the same manner, the used 761 fusion strategy provides good performance for the biometric 762 verification task (EER $=1.10 \%$ in the two sample case). We 763 can note that the adaptation of the algorithms to hardware 764 implementation do not introduce performance degradation. 765 Our experiments demonstrate that the proposed approach is 766 an effective solution for the small sample biometric prob767 lem and can outperform memory-consuming methods, such 768 as the ones that use Gabor filter banks. Moreover, using the 769 same algorithm, performance may be increased with other 770 modalities having an oriented texture such as knuckleprint 771 or ear.

772 This soft- and hardware study shows the feasibility of a 773 robust and efficient embedded multimodal biometric system 774 that offers several advantages; for example, flexibility, user775 friendliness, and real-time processing. Besides, the proposed 776 system is able to work with real-world application challenges, 777 such as lighting changes and variations in hand position and 778 orientation. Our final objective is to implement the complete 779 biometric application on a hardware system. Our next step 780 consists of doing new processing optimizations and com781 plexity analysis of the palmprint image extraction task (hand 782 localization, palmprint extraction and normalization), before 783 achieving FPGA implantations. The chosen FPGA contains a 784 PowerPC processor core that could be used to perform some 785 calculations.

\section{References}

14. A. Poinsot, F. Yang, and M. Paindavoine, "Small sample biometric recognition based on palmprint and face fusion," in ICCGI '09: Proc. of 20094 Int. Multi-Conf. on Computing in the Global Information Technology, p. 118-122 (2009).

15. T. Connie, A. T. B. Jin, M. G. K. Ong, and D. N. C. Ling, "An automated palmprint recognition system," Image Vis. Comput. 23(5), 501-515 (2005).

16. H. Dutagaci, B. Sankur, and E. Yoruk, "Comparative analysis of global hand appearance-based person recognition," J. Electron. Imaging 17(1), (2008).

17. A. Kong, D. Zhang, and M. Kamel, "Palmprint identification using feature-level fusion," Pattern Recogn. 39(3), 478-487 (2006).

18. X. Tan, S. Chen, Z. H. Zhou, and Fuyan Zhang, "Face recognition from a single image per person: a survey," Pattern Recogn. 39(9), 1725-1745 (2006).

19. M. Kyperountas, A. Tefas, and I. Pitas, "Weighted piecewise LDA for solving the small sample size problem in face verification," IEEE Trans. Neural Netw. 18(2), 506-519 (March 2007).

20. D. Xu, S. Yan, L. Zhang, S. Lin, H. J. Zhang, and T. S. Huang, "Reconstruction and recognition of tensor-based objects with concurrent subspaces analysis," IEEE Trans. Circ. Syst. Video Technol. 18(1), 3647 (January 2008).

21. C. Liu and H. Wechsler, "Gabor feature based classification using the enhanced fisher linear discriminant model for face recognition," IEEE Trans. Image Process. 11, 467-476 (2002).

22. O. Ayinde and Y. H. Yang, "Face recognition approach based on rank correlation of gabor-filtered images," Pattern Recogn. 35(6), 1275-1289 (2002).

23. A. Noore, R. Singh, and M. Vatsa, "Robust memory-efficient data level information fusion of multi-modal biometric images," Inf. Fusion 8(4), 337-346 (2007)

24. W. K. Kong, D. Zhang, and W. Li, "Palmprint feature extraction using 2-D gabor filters," Pattern Recogn. 36(10), 2339-2347 (2003).

25. T. Savic and N. Pavesic, "Personal recognition based on an image of the palmar surface of the hand," Pattern Recogn. 40(11), 3152-3163 (2007).

26. Texas instruments, <http://www.ti.com/>.

27. Xilinx, <http://www.xilinx.com/>, (2009).

28. PolyU palmprint database, $<$ http://www.comp.polyu.edu.hk/ > biometrics (2006).

29. A. M. Martinez and R. Benavente, "The AR face database Technical Report No. 24, CVC (june 1998).

30. D. Zhang, W. K. Kong, J. You, and M. Wong, "Online palmprint identification," IEEE Trans. Pattern Anal. Mach. Intell. 25(9), 1041-1050 (2003).

A. K. Jain, A. Ross, and S. Pankanti, "Biometrics: a tool for information security," IEEE Trans. Inf. Forensics Secur. 1(2), 125-143 (2006).

. D. Masip and J. Vitria, "Shared feature extraction for nearest neighbor face recognition," IEEE Trans. Neural Netw. 19(4), 586-595 (April 2008).

3. G. K. O. Michael, T. Connie, and A. B. J. Teoh, "Touch-less palm print biometrics: novel design and implementation," Image Vis. Comput. 26(12), 1551-1560 (2008)

4. J. Doublet, O. Lepetit, and M. Revenu, "Contact less hand recognition using shape and texture features," in Proc. of 8th Int. Conf. on Signal Processing (ICSP'06), Vol. 3, Guilin, China (November 2006).

5. X. Jiang, W. Xu, L. Sweeney, Y. Li, and R. Gross and D. Yurovsky, "New directions in contact free hand recognition," in Proc. IEEE 2 389-392 (September 2007).

6. X. Y. Jing, Y. F. Yao, D. Zhang, J. Y. Yang, and M. Li, "Face and palmprint pixel level fusion and kernel DCV-RBF classifier for small sample biometric recognition," Pattern Recogn. 40(11), 3209-3224 (2007).

7. X. Geng, Z. H. Zhou, and K. Smith-Miles, "Individual stable space: an approach to face recognition under uncontrolled conditions," IEEE Trans. Neural Netw. 19(8), 1354-1368 (2008).

8. S. Zafeiriou, A. Tefas, and I. Pitas, "The discriminant elastic graph matching algorithm applied to frontal face verification," Pattern Recogn. 40(10), 2798-2810 (2007).

9. A. Kumar and D. Zhang, "User authentication using fusion of face and palmprint," Int. J. Image Graph. 9(2), 251-270 (2009).

10. T. Zhang, X. Li, D. Tao, and J. Yang, "Multimodal biometrics using geometry preserving projections," Pattern Recogn. 41(3), 805-813 (2008).

11. F. Yang and M. Paindavoine, "Implementation of an rbf neural network on embedded systems: real-time face tracking and identity verification," IEEE Trans. Neural Netw. 14(5), 1162-1175 (September 2003).

. C. Lopez-Ongil, R. Sanchez-Reillo, J. Liu-Jimenez, F. Casado, L. Sanchez, and L. Entrena, "FPGA implementation of biometric authentication system based on hand geometry," in Proc. of Field Programmable Logic and Application (FPL) Conf. Vol. 3203, p.43-53. (august 2004).

3. J.-H. Yoo, J.-G. Ko, Y.-S. Chung, S.-U. Jung, K.-H. Kim, K.-Y. Moon, and K. Chung, "Design of embedded multimodal biometric systems," in SITIS '07: Proc. of 20073 Int. IEEE Conf. on Signal-Image Technologies and Internet-Based System, Washington, DC, p. 10581062 IEEE Computer Society, (2007).

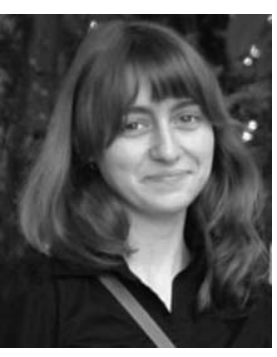

Audrey Poinsot received her MS from 870 the University of Bordeaux I, France in 871 August 2007. Since September 2007, she 872 has been a PhD student in image process- 873 ing and instrumentation at the University of 874 Burgundy. Her research interests consist of 875 multimodal biometrics, with particular em- 876 phasis on their hardware implementations on 877 embedded systems.

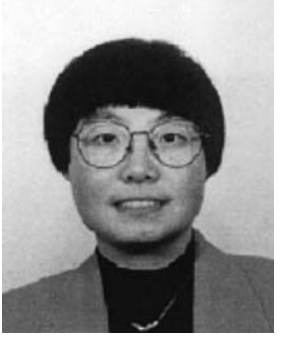

Fan Yang is a full professor and member 879 of LE2I CNRS-UMR, Laboratory of Elec- 880 tronic, Computing, and Imaging Sciences at 88 the University of Burgundy, France. Her re- 882 search interests are in the areas of pat- 883 terns recognition, neural network, motion 884 estimation based on spatiotemporal Gabor 885 filters, parallelism and real-time implementa- 886 tion, and, more specifically, automatic face 887 image-processing algorithms and architectures.

Vincent Brost is an associate professor and member of LE2I CNRSUMR, Laboratory of Electronic, Computing, and Imaging Sciences at 891 the University of Burgundy, France. Before joining the LE2I Labora- 892 tory, he was an engineer in electronics and embedded systems for 893 ten years (for Renault and France Telecom). His research topics are 894 specific processor optimization and real-time hardware implementa- 895 tions on the DSP and the FPGA.

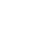

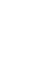




\section{Queries}

Q1: Au: Please check the authors affiliation for correctness.

Q2: Au: Please check Ref. 11 for content errors, or provide the DOI.

Q3: Au: Please provide page range for Ref. 15

Q4: Au: Please check Ref. 20 for content errors, or provide the DOI.

Q5: Au: Please provide date accessed and title document for Ref. 25

Q6: Au: Please supply photograph of author Vincent Brost. 